\title{
Photodynamic Therapy for Halitosis: Could be Possible?
}

\author{
Cinzia Casu $^{1}$ (D), Maria Giulia Nosotti ${ }^{1, *(D)}$ \\ 1 RDH, Departement of Human Science, Innovation and Territory, Dental Hygiene School, University of Insubria, Varese, \\ Italy \\ * Correspondence: ginzia.85@hotmail.it;
}

Scopus Author ID 57195993724

Received: 15.05.2020; Revised: 12.06.2020; Accepted: 15.06.2020; Published: 17.06.2020

\begin{abstract}
Halitosis is a very frequent condition characterized by an unpleasant odor related to expired air, and $80-90 \%$ of the cases originate in the oral cavity. It is a social problem that affects one-third of the population, causing a negative impact on the quality of life. The measurement of volatile sulfur compounds (VSC) at the oral level is very useful for intercepting the problem. Photodynamic Therapy (PDT) is based on the combination of a non-toxic dye and an appropriate wavelength of visible light, which in the presence of oxygen is activated and can promote a phototoxic response. We would like to document a case of oral halitosis diagnosed and treated with photodynamic therapy using a $660 \mathrm{~nm}$ diode laser and phenothiazine chloride as a photosensitizer.
\end{abstract}

Keywords: photodynamic therapy; halitosis; VSC compounds; PDT for halitosis.

(c) 2020 by the authors. This article is an open-access article distributed under the terms and conditions of the Creative Commons Attribution (CC BY) license (https://creativecommons.org/licenses/by/4.0/).

\section{Introduction}

Halitosis is a term used to describe any unpleasant odor related to expired air, a social problem that affects one-third of the population, causing a negative impact on the quality of life. 80 to $90 \%$ of the cases originate in the oral cavity resulting from proteolytic degradation by gram-negative anaerobic bacteria [1,2]. It is mainly caused by the metabolism of the microbiota on the tongue dorsum and periodontium, favored by low salivary flow during the day, food impaction, dental restoration, poor oral hygiene, and diet. Particularly in young patients, the presence of tongue coating seems to be the main cause of halitosis [1].

There are 5 types of halitosis:

Type 1 (oral): this is linked to VSC, volatile organic compounds (VOC), and nitrogencontaining gases (amines) [3]. The principal VSC is hydrogen sulfide (H2S), methyl mercaptans $\left(\mathrm{CH}_{3} \mathrm{SH}\right)$, and dimethyl sulfide $\left(\mathrm{CH}_{3} \mathrm{SCH}_{3}\right)$ [4, 5]. The gases responsible for oral halitosis derive from protein and glycoprotein putrefaction produced by the oral microbiota. The dorsum of the $r$ tongue is the most important halitogenic site, also because it has the largest surface area and the highest bacterial load within a densely populated biofilm [6]. About 85\% of oral halitosis cases are linked to y poor oral hygiene, plaque stagnation areas, and gingivitis/periodontitis.

Type 2 (airway): originates from the respiratory tract (rhinosinusitis, tonsillitis, pharyngitis, laryngitis, bronchitis, pneumonia). The proportion of this type of halitosis is between 2.9 and $10 \%$. Obstructive nasal pathology causes mouth breathing, often resulting in xerostomia and halitosis. Tonsillitis causes edema and hypertrophy, which may obstruct orifices on the tonsillar surface [4]. This fact disrupts the cleansing flow of secretions and desquamated epithelial and bacterial cells, and food debris becomes harnessed, leading to 
stagnation. Anaerobic bacteria found in tonsilloliths include Eubacterium, Fusobacterium, Porphyromonas, Prevotella, Selenomonas, and Tanerella spp., associated with the production of VSCs $[7,8]$

Type 3 (gastroesophageal): this type of halitosis is due to the leakage of odorant volatiles from the stomach through the esophagus to the mouth and nose. A degree of gastroesophageal reflux is considered normal, occurring in almost all individuals several times per day, but pathologically this halitosis occurs due to gastroesophageal reflux disease (GERD), Helicobacter pylori-related gastritis, gastrocolic fistulae and hypopharyngeal diverticula $[9,10]$.

Type 4 (blood-borne): this type of halitosis is due to volatile chemicals in the systemic circulation that $\mathrm{h}$ during alveolar gas exchange move to exhaled breath and cause halitosis. In pathologic Type 4 halitosis exhaled breath volatiles are reported in patients with diabetes mellitus, sleep apnea, H. pylori infection, sickle cell disease, asthma, breast cancer, lung carcinoma, chronic obstructive pulmonary disease, cystic fibrosis, liver diseases, cirrhosis, uremia and kidney failure [11].

Type 5 (subjective): this type can be categorized as psychologic or neurologic [12].

The normal tongue coating is characterized by a thin, slightly moist, and whitish substance. The main factors that affect tongue coating are [13]:

1. the age: In the elderly, the tongue coating is more discolored; this could be due to a modification of the diet, a reduction in salivary flow, or poor oral hygiene.

2. the diet: the tongue coating changes according to the type of food ingested.

3. oral hygiene: this is the main factor that influencing the formation of tongue coating. In particular, patients with periodontal disease may accelerate VSC production; in fact, these patients produced higher concentrations of $\mathrm{CH}_{3} \mathrm{SH}$ than subjects with healthy periodontal tissues, which produced higher concentrations of $\mathrm{H}_{2} \mathrm{~S}$.

The production of VSC metabolites has been considered as the leading cause of intraoral halitosis. Furthermore, short-chain fatty acids (SCFAs), such as propanoic acid and butyric acid, cadaverine, indole, and skatole, may also contribute to the development of halitosis [10].

The sulfur-containing amino acids (cysteine and methionine) are degraded by both Gram-negative and Gram-positive anaerobic bacteria to produce VSCs. A wide range of oral bacteria, including Porphyromonas gingivalis ( $P$. gingivalis), Fusobacterium nucleatum ( $F$. nucleatum), Prevotella intermedia ( $P$. intermedia), Treponema denticola ( $T$. denticola) and Tannerella forsythia ( $T$. forsythia), have been regarded to be closely associated with intraoral halitosis $[14,15]$.

Analysis of VSC is useful for determining levels of $\mathrm{H}_{2} \mathrm{~S}$ and $\mathrm{CH}_{3} \mathrm{SCH}_{3}$ so that both the type of halitosis and the intensity can be assessed [11 ]. The value of $112 \mathrm{ppb} \mathrm{H}_{2} \mathrm{~S}$ indicates the presence of halitosis, while the value of $\mathrm{CH} 3 \mathrm{SH}$ is normal until $26 \mathrm{ppb}$, and this gas is found mainly in periodontal pockets [16].

These bacteria develop mainly on the lingual back, as there are deep fissures in this area that facilitate bacterial proliferation, as they provide a low level of oxygen conditions for halitosis-related anaerobic bacteria. For this reason, the lingual back is considered the main source of VSC production [14]. The process for the acquisition of the sample for the heliometric is based on connecting the device to the computer with a specific software program that allows the creation of a graph corresponding to the peaks and concentrations of VSCs. The 
results are stored in the program recovered when necessary [17]. In order to properly treat halitosis, it is important to detect its source. If it is a bad breath caused by the oral cavity, it is of primary importance to treat any periodontal disease and instruct the patient to maintain correct oral hygiene at home using the brush and interdental aids such as dental floss and pipe cleaner. Sometimes, however, halitosis can occur even without the presence of periodontal disease; for this reason, it is good practice to instruct patients to clean the tongue daily using the brush and tongue cleaner. However, there are other conditions that can lead to the development of halitosis, such as old restorations, unsuitable prostheses, xerostomia, pericoronitis, oral ulceration, or other types of lesions. First of all, therefore, it is essential to diagnose the cause, treat it and, as the last step, treat halitosis [18].

If instead, the halitosis originates from nonoral causes such as respiratory, gastrointestinal and hepatic, renal, endocrine, or hematological disease, consultation should be done with the specialist. Finally, some people think they have halitosis (halitophobia) even if it is not detected during the diagnosis. In severe cases, these subjects must be treated from a psychological point of view [19].

In addition to mechanical treatment, chemical agents, such as mouthwashes, can be used to help combat halitosis, in particular: chlorhexidine, essential oils, triclosan, hydrogen peroxide, oxidizing lozenges, baking soda, Amine fluoride/Stannous fluoride [20].

Another treatment which, according to the data reported in the literature, can generate good results is photodynamic therapy (PDT). This aid has been widely employed in dentistry, mainly for the decontamination of root canals and periodontal pockets [21, 22]. It is based on the combination of a non-toxic dye and an appropriate wavelength of visible light, which in the presence of oxygen is activated and can promote a phototoxic response. These factors, combined together, can produce lethal cytotoxic ROS that can selectively destroy cells. ROS can cause cell death through several mechanisms, including lipid peroxidation, inhibition of enzymatic systems, and agglutination of proteins. PDT can be repeated several times since it is a non-invasive procedure [21].

Studies in the literature have shown that PDT for the treatment of halitosis is effective, without being an invasive treatment. Through this treatment, the particular reduction of $\mathrm{H} 2 \mathrm{~S}$ $[23,24]$ was found. According to the study of Costa de Mota et al., is found a $7 \%$ reduction in bacterial load in the PDT treatment group while reporting a 7\% increase for the group that had used only the lingual scraper.

Furthermore, the study by Lui et al. reported that the use of PDTs with methylene blue and wavelengths between 635 and $670 \mathrm{~nm}$ reduces the levels of $P$. gingivalis, $T$. forsythensis e T. denticola [25].

Although PDT leads to good results in reducing the bacterial load on the lingual back and therefore reducing halitosis, the best results in the literature are reported by the synergy between the PDT and the lingual scraper [26].

We would like to document a case of oral halitosis treated with photodynamic therapy.

\section{Materials and Methods}

A 54 years old man went to our private practice for a normal follow up. He reported good general health and that to have had the sensation of bad breath in the mouth. The patient had good oral hygiene, and he reported to clean every day the tongue. It was therefore decided to carry out a photodynamic therapy session with $660 \mathrm{~nm}$ diode laser, 100 mwatt of power, a spot of $1 \mathrm{~cm}$ (Helbo, Bredent, Senden, Germany) and phenothiazine chloride 0,1\%. This 
photosensitizer has been spread along the lingual surface, especially on the posterior side. After 1 minute, the laser light had been activated, with a 2D tip, maintained approximately $0.5 \mathrm{~cm}$ from the tongue and making circular movements for a total of 5 minutes (fig 1). Subsequently, the photosensitizer was removed with a cotton ball soaked with a physiological solution 1 .

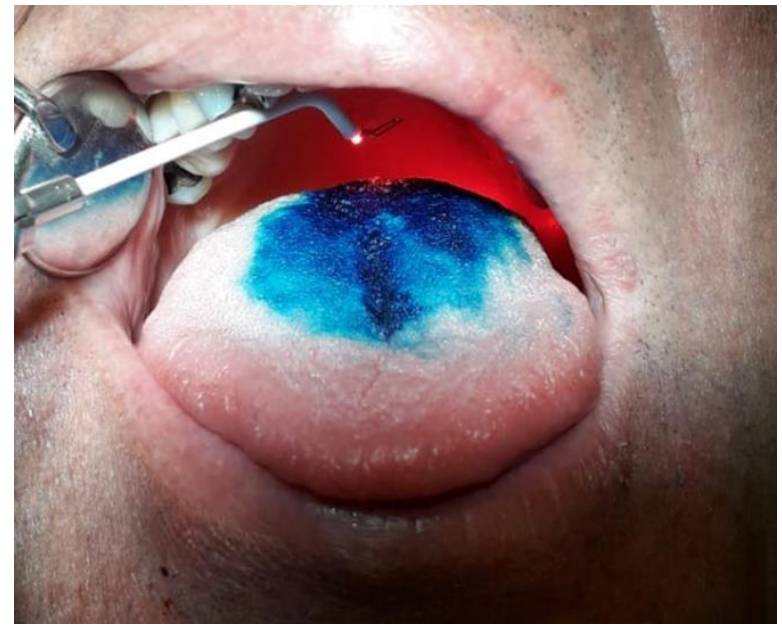

Figure 1. Tongue of the patient during Photodynamic Therapy.

\section{Results and Discussion}

After a week, the patient felt an improvement in symptoms, and the same results were maintained at 1 month follow up. It is very interesting to evaluate also VSC values. We cannot determine whether the patient improved his condition also for greater lingual cleansing or whether he affected his post-treatment diet. Halitosis plays an important role in the quality of life; for this reason, it is a topic that needs attention to avoid the patient's social isolation. It is a condition that mainly involves anaerobic bacteria, which release VSC during exhalation, are found mainly on the lingual back, and are the cause of the bad smell [27]. According to the literature, halitosis can arise more easily in subjects with poor oral hygiene, wearers of incongruous prostheses, and old restorations. In particular, in elderly subjects wearing prostheses, the correct hygienic maintenance of prosthetic products can be difficult, leading to bacterial colonization, and the onset of bad odors [28].

Therefore, the need for new therapeutic alternatives for the patient is fundamental, as studies have shown that self-cleaning of the tongue alone, using tongue scrapers, is not completely effective for the reduction of halitosis.

Furthermore, the use of mouthwashes as an antimicrobial action is also not completely effective, as they do not eliminate the cause and also cause side effects such as unpleasant taste, alteration of the oral microflora, and coloring of the mucous membranes.

The study conducted by Seerangaiyan et al., analyzed the quality of the tongue microbial in subjects with and without halitosis. From what can be deduced, the test group (with halitosis) presented Aggregatibacter segnis, Campylobacter, Capnocytophaga, Clostridiales, Dialister, Leptotrichia, Parvimonas, Peptostreptocococcus, Peptococcus, Prevotella, Selenomonas, Treannerella, while the control group had (without) Haemophilus parainfluenza (H. parainfluenza), Moryella, Oribacterium, Prevotella, several Streptococcus, Rothia dentocariosa [29, 30].

Furthermore, from the meta-analysis conducted by Dou et al., it is shown that H. pylori infection is related to halitosis. This data can be useful to treat those patients refractory to therapies [31]. 
PDT, according to the literature, has proven to be a valid alternative, it is also a noninvasive procedure, does not cause side effects, does not cause bacterial resistance, and the effects are immediately visible [6].

Gonçalves' study assessed the presence of halitosis in patients with multiple sclerosis, who showed higher levels of $\mathrm{H}_{2} \mathrm{~S}$, compared to the control group not affected by multiple sclerosis. These subjects could be more predisposed to the formation of $\mathrm{H}_{2} \mathrm{~S}$ for the intake of immunosuppressants, which could alter the salivary flow and, therefore, the oral flora. In this study, it was reported that the combination of the lingual scraper with PDT is more effective in reducing the concentration of VSC than using only the tongue scraper or only the PDT [1].

Another study conducted by Costa da Mota et al., evaluated the clinical and microbiological effect of antimicrobial photodynamic therapy (aPDT) on halitosis in adolescents. They were divided into three groups: group 1 (treatment with photodynamic therapy), group 2 (treatment with tongue scraper), group 3 (treatment with a tongue scraper, and photodynamic therapy). The results reported that the administration of photodynamic therapy alone reduces the concentration of $\mathrm{H}_{2} \mathrm{~S}$, therefore of halitosis, compared to the use of tongue scrapers only [26].

A further study, conducted by Garcia Lopes et al., divided adolescents into three groups: group 1 (treatment with photodynamic therapy), group 2 (treatment with a tongue scraper), group 3 (treatment with tongue scraper combined with photodynamic therapy). This study shows that the greatest reduction in sulfides occurs by combining photodynamic therapy with the use of the tongue scraper. [6]. All the data provided in the literature report that photodynamic therapy combined with the correct deduction of the tongue drastically reduces the concentration level of VSC, in particular $\mathrm{H}_{2} \mathrm{~S}$, compared to just cleansing the tongue or using antimicrobial agents $[32,33]$.

\section{Conclusions}

Phenothiazine derivatives such as methylene blue are very used in the field of chemistry, biology, and pharmacology [34]. In our case, we have used a phenothiazine derivative, and the success of this photosensitizer is well documented in the recent literature $[35,36,37]$. For this reason, the photodynamic therapy treatment can be recommended in halitosis patients, and also, it can be carried out several times as it is a non-invasive treatment capable of immediately generating benefits.

\section{Funding}

This research received no external funding.

\section{Acknowledgments}

The authors declare no acknowledgment.

\section{Conflicts of Interest}

The authors declare no conflict of interest. 


\section{References}

1. Gonçalves, M.L.L.; Kalil Bussadori, S.; Dadalti Fragoso, Y.; da Silva, V.V.B.; Melo Deana, A.; da Mota, A.C.C.; Horácio Pinto, E.; Horliana, A.C.R.; Miranda França, C. Effect of photodynamic therapy in the reduction of halitosis in patients with multiple sclerosis: clinical trial. Journal of Breath Research 2017, 11.

2. Silva, M.F.; Leite, F.R.M.; Ferreira, L.B.; Pola, N.M.; Scannapieco, F.A.; Demarco, F.F.; Nascimento, G.G. Estimated prevalence of halitosis: a systematic review and meta-regression analysis. Clin Oral Investig 2018, 22, 47-55, https://doi.org/10.1007/s00784-017-2164-5.

3. Aydin, M.; Harvey-Woodworth, C.N. Halitosis: a new definition and classification. British Dental Journal 2014, 217, E1-E1, https://doi.org/10.1038/sj.bdj.2014.552.

4. Lopes, R.G.; de Godoy, C.H.L.; Deana, A.M.; de Santi, M.E.S.O.; Prates, R.A.; França, C.M.; Fernandes, K.P.S.; Mesquita-Ferrari, R.A.; Bussadori, S.K. Photodynamic therapy as a novel treatment for halitosis in adolescents: study protocol for a randomized controlled trial. Trials 2014, 15, 443-443, https://doi.org/10.1186/1745-6215-15-443.

5. Sombie, R.; Tiendrebeogo, A.J.F.; Guiguimde, W.P.L.; Guingane, A.; Tiendrebeogo, S.; Ouoba, K.; Bougouma, A. Halitosis: multidisciplinary diagnostic and therapeutic approaches. Pan Afr Med J 2018, 30, 201, https://doi.org/10.11604/pamj.2018.30.201.10951.

6. Lopes, R.G.; da Mota, A.C.C.; Soares, C.; Tarzia, O.; Deana, A.M.; Prates, R.A.; França, C.M.; Fernandes, K.P.S.; Ferrari, R.A.M.; Bussadori, S.K. Immediate results of photodynamic therapy for the treatment of halitosis in adolescents: a randomized, controlled, clinical trial. Lasers in Medical Science 2016, 31, 41-47, https://doi.org/10.1007/s10103-015-1822-6.

7. Riggio, M.P.; Lennon, A.; Rolph, H.J.; Hodge, P.J.; Donaldson, A.; Maxwell, A.J.; Bagg, J. Molecular identification of bacteria on the tongue dorsum of subjects with and without halitosis. Oral Diseases 2008, 14, 251-258, https://doi.org/10.1111/j.1601-0825.2007.01371.x.

8. Nakhleh, M.K.; Quatredeniers, M.; Haick, H. Detection of halitosis in breath: Between the past, present, and future. Oral Diseases 2018, 24, 685-695, https://doi.org/10.1111/odi.12699.

9. Kapoor, U.; Sharma, G.; Juneja, M.; Nagpal, A. Halitosis: Current concepts on etiology, diagnosis and management. Eur J Dent 2016, 10, 292-300, https://doi.org/10.4103/1305-7456.178294.

10. Anbari, F.; Ashouri Moghaddam, A.; Sabeti, E.; Khodabakhshi, A. Halitosis: Helicobacter pylori or oral factors. Helicobacter 2019, 24, e12556, https://doi.org/10.1111/hel.12556.

11. Bicak, D.A. A Current Approach to Halitosis and Oral Malodor- A Mini Review. Open Dent J 2018, 12, 322330, https://doi.org/10.2174/1874210601812010322.

12. Vali, A.; Roohafza, H.; Hassanzadeh Keshteli, A.; Afghari, P.; Javad Shirani, M.; Afshar, H.; Savabi, O.; Adibi, P. Relationship between subjective halitosis and psychological factors. International Dental Journal 2015, 65, 120-126, https://doi.org/10.1111/idj.12153.

13. Seerangaiyan, K.; Juch, F.; Winkel, E.G. Tongue coating: its characteristics and role in intra-oral halitosis and general health-a review. J Breath Res 2018, 12

14. Ye, W.; Zhang, Y.; He, M.; Zhu, C.; Feng, X.P. Relationship of tongue coating microbiome on volatile sulfur compounds in healthy and halitosis adults. J Breath Res 2019, 14, https://doi.org/10.1088/1752$7163 / \mathrm{ab} 47 \mathrm{~b} 4$.

15. Wu, X.; Zhang, J.; Zhou, Y.; He, Z.; Cai, Q.; Nie, M. Whether Chinese Medicine Have Effect on Halitosis: A Systematic Review and Meta-Analysis. Evidence-Based Complementary and Alternative Medicine 2018, 2018, https://doi.org/10.1155/2018/4347378

16. Suzuki, N.; Yoneda, M.; Takeshita, T.; Hirofuji, T.; Hanioka, T. Induction and inhibition of oral malodor. Mol Oral Microbiol 2019, 34, 85-96, https://doi.org/10.1111/omi.12259.

17. Lopes, R.G.; de Santi, M.E.; Franco, B.E.; Deana, A.M.; Prates, R.A.; Franca, C.M.; Fernandes, K.P.; Ferrari, R.A.; Bussadori, S.K. Photodynamic therapy as novel treatment for halitosis in adolescents: a case series study. J Lasers Med Sci 2014, 5, 146-152.

18. Torsten, M.; Gomez-Moreno, G.; Aguilar-Salvatierra, A. Drug-related oral malodour (halitosis): a literature review. Eur Rev Med Pharmacol Sci 2017, 21, 4930-4934.

19. Aylikci, B.; Çolak, H. Halitosis: From diagnosis to management. Journal of Natural Science, Biology and Medicine 2013, 4, 14-23, https://doi.org/10.4103/0976-9668.107255.

20. De Geest, S.; Laleman, I.; Teughels, W.; Dekeyser, C.; Quirynen, M. Periodontal diseases as a source of halitosis: a review of the evidence and treatment approaches for dentists and dental hygienists. Periodontology 2000 2016, 71, 213-227, https://doi.org/10.1111/prd.12111. 
21. Carrera, E.T.; Dias, H.B.; Corbi, S.C.T.; Marcantonio, R.A.C.; Bernardi, A.C.A.; Bagnato, V.S.; Hamblin, M.R.; Rastelli, A.N.S. The application of antimicrobial photodynamic therapy (aPDT) in dentistry: a critical review. Laser Phys 2016, 26, https://doi.org/10.1088/1054-660X/26/12/123001.

22. Kwiatkowski, S.; Knap, B.; Przystupski, D.; Saczko, J.; Kędzierska, E.; Knap-Czop, K.; Kotlińska, J.; Michel, O.; Kotowski, K.; Kulbacka, J. Photodynamic therapy - mechanisms, photosensitizers and combinations. Biomedicine \& Pharmacotherapy 2018, 106, 1098-1107, https://doi.org/10.1016/j.biopha.2018.07.049.

23. Costa da Mota, A.C.; França, C.M.; Prates, R.; Deana, A.M.; Costa Santos, L.; Lopes Garcia, R.; Leal Gonçalves, M.L.; Mesquita Ferrari, R.A.; Porta Santos Fernandes, K.; Kalil Bussadori, S. Effect of photodynamic therapy for the treatment of halitosis in adolescents - a controlled, microbiological, clinical trial. Journal of Biophotonics 2016, 9, 1337-1343, https://doi.org/10.1002/jbio.201600067.

24. Maehara, S.; Norihiko Ikeda, N. Photodynamic Therapy. Kyobu Geka 2018, 71, 858-861.

25. Lui, J.; Corbet, E.F.; Jin, L. Combined photodynamic and low-level laser therapies as an adjunct to nonsurgical treatment of chronic periodontitis. $J$ Periodontal Res 2011, 46, 89-96, https://doi.org/10.1111/j.1600-0765.2010.01316.x.

26. Costa da Mota, A.C.; Franca, C.M.; Prates, R.; Deana, A.M.; Costa Santos, L.; Lopes Garcia, R.; Leal Goncalves, M.L.; Mesquita Ferrari, R.A.; Porta Santos Fernandes, K.; Kalil Bussadori, S. Effect of photodynamic therapy for the treatment of halitosis in adolescents - a controlled, microbiological, clinical trial. J Biophotonics 2016, 9, 1337-1343, https://doi.org/10.1002/jbio.201600067.

27. Goncalves, M.L.L.; da Mota, A.C.C.; Deana, A.M.; Guedes, G.H.; Cavalcante, L.A.S.; Prates, R.A.; Horliana, A.; Pavani, C.; Motta, L.J.; Bitencourt, G.B.; Fernandes, K.P.S.; Salgueiro, M.; Mesquita-Ferrari, R.A.; da Silva, D.F.T.; Franca, C.M.; Bussadori, S.K. Photodynamic therapy with Bixa orellana extract and LED for the reduction of halitosis: study protocol for a randomized, microbiological and clinical trial. Trials 2018, 19, 590, https://doi.org/10.1186/s13063-018-2913-z.

28. Vale, K.L.D.; Horliana, A.; Romero, S.D.S.; Deana, A.M.; Goncalves, M.L.L.; Ferrari, R.A.M.; Bussadori, S.K.; Fernandes, K.P.S. Evaluation of the treatment of halitosis with photodynamic therapy in older patients with complete denture: Protocol for a randomized, controlled trial. Medicine (Baltimore) 2019, 98 , https://doi.org/10.1097/md.0000000000016275.

29. Seerangaiyan, K.; Juch, F.; Winkel, E.G. Tongue coating: its characteristics and role in intra-oral halitosis and general health-a review. $J$ Breath Res 2018, 12.

30. Perez-Laguna, V.; Garcia-Malinis, A.J.; Aspiroz, C.; Rezusta, A.; Gilaberte, Y. Antimicrobial effects of photodynamic therapy. G Ital Dermatol Venereol 2018, 153, 833-846, https://doi.org/10.23736/S03920488.18.06007-8.

31. Dou, W.; Li, J.; Xu, L.; Zhu, J.; Hu, K.; Sui, Z.; Wang, J.; Xu, L.; Wang, S.; Yin, G. Halitosis and helicobacter pylori infection: A meta-analysis. Medicine 2016, 95, e4223-e4223, https://doi.org/10.1097/MD.0000000000004223.

32. Rai, M.; Spratt, D.; Gomez-Pereira, P.R.; Patel, J.; Nair, S.P. Light activated antimicrobial agents can inactivate oral malodour causing bacteria. J Breath Res 2016, 10, https://doi.org/10.1088/17527155/10/4/046009.

33. Romero, S.D.S.; Schalch, T.O.; do Vale, K.L.; Ando, E.S.; Mayer, M.P.A.; Feniar, J.P.G.; Fernandes, K.P.S.; Bussadori, S.K.; Motta, L.J.; Negreiros, R.M.; Tempestini Horliana, A.C.R. Evaluation of halitosis in adult patients after treatment with photodynamic therapy associated with periodontal treatment: Protocol for a randomized, controlled, single-blinded trial with 3-month follow up. Medicine 2019, 98, https://doi.org/10.1097/MD.0000000000016976.

34. Al-Anber, M.A.; Almogbel, M.S.; Al-Anber, Z.A. Sorption of methylene blue onto the biomass of date palm seeds: Kinetic study. Biointerface Research in Applied Chemistry 2018, 8, 3583-3589.

35. Santos, D.d.A.; Crugeira, P.J.L.; Nunes, I.P.F.; de Almeida, P.F.; Pinheiro, A.L.B. A novel technique of antimicrobial photodynamic therapy - aPDT using 1,9-dimethyl-methylene blue zinc chloride double saltDMMB and polarized light on Staphylococcus aureus. Journal of Photochemistry and Photobiology B: Biology 2019, 200, https://doi.org/10.1016/j.jphotobiol.2019.111646.

36. Poli, P.P.; Souza, F.Á.; Ferrario, S.; Maiorana, C. Adjunctive application of antimicrobial photodynamic therapy in the prevention of medication-related osteonecrosis of the jaw following dentoalveolar surgery: A case series. Photodiagnosis and Photodynamic Therapy 2019, 27, 117-123, https://doi.org/10.1016/j.pdpdt.2019.05.037. 
37. Ciarcia, A.; Goncalves, M.L.L.; Horliana, A.; Suguimoto, E.S.A.; Araujo, L.; Laselva, A.; Mayer, M.P.A.; Motta, L.J.; Deana, A.M.; Mesquita-Ferrari, R.A.; Fernandes, K.P.S.; Bussadori, S.K. Action of antimicrobial photodynamic therapy with red leds in microorganisms related to halitose: Controlled and randomized clinical trial. Medicine (Baltimore) 2019, 98, e13939, https://doi.org/10.1097/md.0000000000013939. 